\title{
100 years young
}

\section{This is the International Year of Crystallography and is a time to reflect on the success of the discipline. Looking ahead the field is well placed to produce further exciting contributions to science.}

Our print subscribers will find a pleasant surprise this month. Along with their August issue of Nature Nanotechnology there comes a booklet entitled Nature Milestones in Crystallography. These extra 42 pages recount the history of crystallography in 25 defining moments and contains reprints of 8 breakthrough communications and papers from the Nature archives. (Online readers can find the Milestones in Crystallography at http://www.nature.com/milestones/ milecrystal/index.html; the content is free for 6 months and contains an online-only extensive collection of articles published across Nature-branded journals starting with an 1883 paper by William Barlow on the packing of hard spheres.)

The origins of modern crystallography can be traced back to Max Laue who, against the backdrop of pre-First World War Germany, conceived what Albert Einstein had no hesitation labelling as one of the most beautiful experiments in physics: the diffraction of X-rays by a crystal lattice. The implications were immediately evident to scientists of the time and in just a few years a new tool for the investigation of matter with atomic precision was developed (mainly courtesy of William Lawrence and William Henry Bragg).

Crystallography usually provides conclusive results. The planarity of benzene, the existence of ionic crystals and of sandwich compounds such as ferrocene, and the determination of complex biological structures are but a few examples in which crystallography was able to solve scientific controversies once and for all. As a consequence, crystallography has been adopted across the sciences right from the start.

The relationship between crystallography and nanoscale objects can be traced back to the discovery of powder diffraction by Paul Scherrer and his formula to measure the size of colloidal particles. A hundred years later, researchers in nanoscience enjoy a set of crystallographic techniques specifically tailored for their sample conditions. Large-scale facilities offer sophisticated tools that can, for example, access the physicochemical properties of nanometre-thick films and even monolayers. And there are no signs of X-ray, neutron or electron crystallography slowing down, whether they be used for scattering, spectroscopy or imaging. In particular, the fourth generation of synchrotron radiation facilities is expected to deliver an

Short pulse duration also means that it will be possible to detect diffraction signals before the sample is irreversibly damaged. This will allow researchers to determine the structure of single macromolecules and carry out measurements in operando conditions, for example, following the motion of charge carriers directly in a device, or studying single events occurring at the electrode interface in energy-conversion and energy-storage devices. Overall, the next

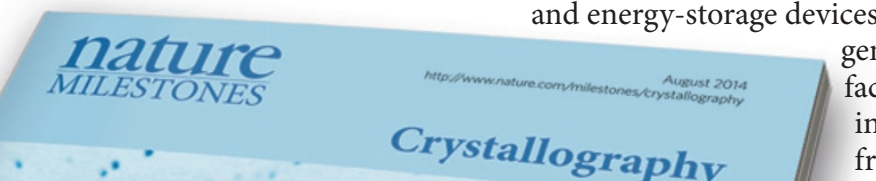
generation of X-ray facilities, which includes X-ray free-electron lasers, and the largely complementary neutron scattering techniques will allow researchers to gain a stronger connection between nanoscale structure and macroscopic function of matter. Moreover, with the ability to focus beams in increasingly tighter regions, it will be possible to induce specific transformations with nanometre-scale spatial resolution, which could be useful for triggering specific self-assembly behaviours or building multicomponent devices. Furthermore, imaging techniques that can already reach respectable resolutions are expected to achieve resolutions of less than 1 nanometre.

We hope that while going through our Milestone, readers will marvel at how far the field has come

understanding of matter with unprecedented resolution and add a whole new dimension to experiments: that of dynamics, thanks to brilliant subfemtosecond pulses. This capability should be exciting news for nanoscience.

With such methods, it will be possible to study single nanoparticles and characterize, for instance, the role of defects during growth, or the catalytic properties of the surface by 'watching' reactions in real time. in 100 years and be inspired to imagine what crystallography can do for them.

Nanotechnology is, after all, about control at the smallest possible scale, and crystallography has the potential to offer the enabling tools.

Editorially, Milestones in Crystallography has been a cross-journal collaboration between Nature Nanotechnology, Nature Materials and Nature Molecular \& Structural Biology, reflecting the interdisciplinary character of the field. 